\title{
Hipotermia na circulação extracorpórea em cirurgia cardíaca
}

\author{
Hypothermia in extracorporeal circulation in cardiac surgery \\ Hipotermia en la circulación extracorpórea en cirugía cardíaca
}

Recebido: 07/12/2020 | Revisado: 12/12/2020 | Aceito: 11/03/2021 | Publicado: 18/03/2021

Tiago de Castro Carneiro

ORCID: https://orcid.org/0000-0002-2262-7168 Instituto Nacional de Cardiologia, Brasil

E-mail: tiagodcc@hotmail.com

\begin{abstract}
Resumo
Este artigo tem como objetivo compreender a importância da hipotermia durante a circulação extracorpórea. Surge com a premissa de entender a importância da hipotermia induzida na realização da cirurgia cardíaca com Circulação Extracorpórea (CEC), contudo, se faz necessário compreender que o surgimento da CEC na cirurgia cardíaca foi um marco para história da saúde, permitindo a manipulação direta do coração em muitas patologias até então consideradas incuráveis. Utilizou-se como metodologia revisão integrativa, com busca em artigos existentes na literatura. A busca dos artigos foi realizada entre os meses de junho e agosto de 2020. Para o estudo, utilizou-se artigos nacionais publicados na íntegra, adotou-se a estratégia de utilizar cada descritor isoladamente associado aos filtros de interesse disponíveis na Biblioteca Virtual em Saúde (BVS), com as bases de dados LILACS (Literatura Latino-americana e do Caribe em Ciências da Saúde) e SCIELO (Scientific Electronic Library Online), utilizando os seguintes descritores: Hipotermia, Circulação Extracorpórea, Cirurgia Cardíaca. Identificamos um ponto importante a qual esta pesquisa corrobora, mostrando que o controle da temperatura durante o processo operatório, tanto no período de hipotermia, se faz necessário para diminuir o metabolismo. A hipotermia é um procedimento que transcende a nossa expectativa, este possibilita parar totalmente a circulação por uma hora ou mais, e posteriormente ao aquecimento, ver o paciente voltar ao seu estado de metabolismo homeotérmico e à vida. Este estudo permitiu uma reflexão a atuação nas cirurgias cardíaca, difundindo o conhecimento do uso da hipotermia neste tipo de cirurgia. Concluindo que apesar de muitos avanços tecnológicos, a hipotermia ainda persiste em não ser utilizada para evitar as complicações fisiopatológicas. Sendo assim, indispensável para descrever os mecanismos que são positivos utilizados na hipotermia.
\end{abstract}

Palavras-chave: Hipotermia; Circulação extracorpórea; Cirurgia cardíaca.

\begin{abstract}
This article aims to understand the importance of hypothermia during cardiopulmonary bypass. It emerges with the premise of understanding the importance of hypothermia induced in performing cardiac surgery with cardiopulmonary bypass (CPB), however, it is necessary to understand that the emergence of CPB in cardiac surgery was a milestone in the history of health, allowing the direct manipulation of the heart in many pathologies hitherto considered incurable. An integrative review methodology was used, with a search for existing articles in the literature. The search for articles was carried out between the months of June and August 2020. For the study, national articles published in full were used, the strategy of using each descriptor in isolation associated with the filters of interest available in the Virtual Health Library was adopted. (VHL), with LILACS (Latin American and Caribbean Literature in Health Sciences) and SCIELO (Scientific Electronic Library Online) databases, using the following descriptors: Hypothermia, Extracorporeal Circulation, Cardiac Surgery. We have identified an important point that this research supports, showing that temperature control during the operative process, both in the hypothermia period, is necessary to decrease metabolism. Hypothermia is a procedure that transcends our expectation, this makes it possible to stop circulation completely for an hour or more, and after warming up, see the patient return to his state of homeothermal metabolism and life. This study allowed a reflection on the performance of cardiac surgeries, disseminating knowledge about the importance of using hypothermia in this type of surgery. In conclusion, despite many technological advances, hypothermia still persists in not being used to avoid pathophysiological complications. Therefore, it is essential to describe the mechanisms that are positive in the use of hypothermia.
\end{abstract}

Keywords: Hypothermia; Extracorporeal circulation; Cardiac surgery.

Resumen

Este artículo tiene como objetivo comprender la importancia de la hipotermia durante el bypass cardiopulmonar. Surge con la premisa de comprender la importancia de la hipotermia inducida en la realización de cirugía cardíaca con circulación extracorpórea (CEC), sin embargo, es necesario entender que la aparición de la CEC en cirugía cardíaca fue un hito en la historia de la salud, permitiendo la manipulación directa de la corazón en muchas patologías hasta ahora consideradas incurables. Se utilizó una metodología de revisión integradora, con búsqueda de artículos 
existentes en la literatura. La búsqueda de artículos se realizó entre los meses de junio y agosto de 2020. Para el estudio se utilizaron artículos nacionales publicados íntegramente, se adoptó la estrategia de utilizar cada descriptor de forma aislada asociado a los filtros de interés disponibles en la Biblioteca Virtual en Salud. (BVS), con las bases de datos LILACS (Literatura Latinoamericana y Caribeña en Ciencias de la Salud) y SCIELO (Scientific Electronic Library Online), utilizando los siguientes descriptores: Hipotermia, Circulación Extracorpórea, Cirugía Cardiaca. Hemos identificado un punto importante que respalda esta investigación, mostrando que el control de la temperatura durante el proceso operatorio, tanto en el período de hipotermia, es necesario para disminuir el metabolismo. La hipotermia es un procedimiento que trasciende nuestras expectativas, esto permite detener la circulación por completo durante una hora o más, y luego del calentamiento, ver al paciente regresar a su estado de vida y metabolismo homeotermal. Este estudio permitió reflexionar sobre la realización de las cirugías cardíacas, difundiendo conocimientos sobre la importancia del uso de la hipotermia en este tipo de cirugías. En conclusión, a pesar de muchos avances tecnológicos, la hipotermia aún persiste en no ser utilizada para evitar complicaciones fisiopatológicas. Por tanto, es fundamental describir los mecanismos positivos en el uso de la hipotermia.

Palabras clave: Hipotermia; Circulación extracorpórea; Cirugía cardíaca.

\section{Introdução}

Este estudo surge com a premissa de entender a importância da hipotermia induzida na realização da cirurgia cardíaca com Circulação Extracorpórea (CEC), contudo, se faz necessário compreender que o surgimento da CEC na cirurgia cardíaca foi um marco para história da saúde, permitindo a manipulação direta do coração em muitas patologias até então consideradas incuráveis. No Brasil em outubro de 1955 foi efetuado a primeira cirurgia cardíaca utilizando a CEC, onde o Professor Hugo João Felipozzi o responsável pela primeira máquina de CEC (Freitas, Santos \& Oliveira, 2017; Pereira, 2013).

A circulação artificial foi introduzida em laboratório por Gibbon em 1935, onde ocorreu à primeira aplicação clínica da CEC em 1951, sua segunda aplicação clínica em 1953, Gibbon então encerrou com sucesso uma comunicação interauricular, utilizando o seu desenho de máquina coração-pulmão $(\mathrm{CP})$, o que veio a desencadear a revolução da cirurgia cardíaca atual. Assim a CEC foi sendo aperfeiçoada com uma evolução considerável nos últimos 60 anos, contribuindo para redução da morbimortalidade na cirurgia cardíaca. Se faz necessário ate os dias atuais a redução de complicações em especial as neurológicas, que representam como a causa principal de mortalidade no período pós-operatório (Freitas, Santos \& Oliveira, 2017).

Abreu (2014) e Freitas, Santos e Oliveira (2017), citam que o procedimento de CEC, é utilizada em diferentes tipos de cirurgia cardíaca. Esta tem por finalidade, ofertar um coração móvel e sem sangue para melhor realização da cirurgia cardíaca proposta, enquanto os diferentes sistemas orgânicos permanecem perfundidos com sangue oxigenado, preservando as funções cardíacas. Neste procedimento o sangue passa por um aparelho de derivação cardiopulmonar, com a CEC o coração e os pulmões se excluem por completo da circulação. Segundo Abreu (2014) e Dienstmann, Caregnato (2013), existe a necessidade da utilização de heparina, hemodiluição, um certo grau de hipotermia e uma bomba externa para que se impulsione o fluxo sanguíneo. Isso ocorre porque o sangue na CEC encontra-se em contato com superfícies não biológicas

Para compreensão deste estudo, é necessário entender o que seria a hipotermia, que seria um procedimento com a aplicação terapêutica do frio, realizando a diminuição da temperatura corporal abaixo de $35^{\circ} \mathrm{C}$, esta não poderá chegar a $32^{\circ} \mathrm{C}$. Esta terapêutica consiste em diminuir a demanda de oxigênio, exercendo a proteção dos órgãos vitais (cérebro, coração, rins), ainda diminui a frequência cardíaca e melhora a perfusão miocárdica (Barbosa, Cardinelli \& Ercole, 2010; Longo, 2011).

No entanto, o estudo tem como objetivo geral: compreender a importância da hipotermia durante a circulação extracorpórea. E os objetivos específicos: avaliar os benefícios da hipotermia na circulação extracorpórea na cirurgia cardíaca; comparar a eficácia da hipotermia e da normotermia na CEC; registrar os achados na literatura dos benefícios da hipotermia na circulação extracorpórea. 


\section{Metodologia}

Este estudo é uma revisão integrativa, com busca em artigos presentes na literatura. A busca dos artigos foi realizada entre os meses de junho e agosto de 2020. Neste estudo, utilizou-se artigos nacionais publicados na íntegra, adotou-se a estratégia de utilizar cada descritor isoladamente associado aos filtros de interesse disponíveis na Biblioteca Virtual em Saúde (BVS), com as bases de dados LILACS (Literatura Latino-americana e do Caribe em Ciências da Saúde) e SCIELO (Scientific Electronic Library Online), utilizando os seguintes descritores: Hipotermia, Circulação Extracorpórea, Cirurgia Cardíaca.

Os critérios de inclusão utilizados foram: artigos na íntegra, nos últimos 8 anos (2011-2019), com o refinamento utilizando os 3 descritores hipotermia, circulação extracorpórea, cirurgia cardíaca, foram selecionados 40 artigos científicos. Os critérios de exclusões foram: artigos que não eram relacionados ao tema e idioma que não o português. Após a leitura na íntegra dos artigos selecionados para os resultados, estes foram categorizados conforme seus resultados. Desta forma foram selecionados 14 artigos para construção do artigo.

\section{Resultados e Discussão}

No entanto, este estudo teve como amostragem final da revisão integrativa de literatura, esta constituída por 14 artigos científicos, selecionados pelos critérios de inclusão e exclusão previamente estabelecidos. No quadro 1, apresenta as especificações dos artigos.

Quadro 1. Distribuição de publicações segundo ano de publicação, autor, título e periódico de publicação.

\begin{tabular}{|c|c|c|c|c|}
\hline $\begin{array}{c}\text { Ano de } \\
\text { publicação }\end{array}$ & Autor & Título & Periódico de publicação & Ordem \\
\hline 2014 & Thiago Titonel Abreu & $\begin{array}{l}\text { Análise da variação de } \\
\text { eletrólitos após cirurgia } \\
\text { cardíaca com uso de } \\
\text { circulação extracorpórea. }\end{array}$ & $\begin{array}{l}\text { UFBA/SIBI/Biblioteca } \\
\text { Gonçalo Moniz: Memória } \\
\text { da Saúde Brasileira }\end{array}$ & 1 \\
\hline 2010 & $\begin{array}{l}\text { Natia de Freitas Barbosa, } \\
\text { Danilo Martins Cardinelli, } \\
\text { Flávia Falci Ercole. }\end{array}$ & $\begin{array}{l}\text { Determinantes } \\
\text { Complicações de } \\
\text { no Uso da Cirológicas } \\
\text { Extracorpórea }(\mathrm{CEC}) .\end{array}$ & $\begin{array}{ll}\text { Arquivo } & \text { Brasileiro } \\
\text { Cardiologia } & \end{array}$ & 2 \\
\hline 2019 & $\begin{array}{l}\text { Sandy Ribeiro Barros; } \\
\text { Michele Matias Bandeira; } \\
\text { Jandra Cibele R. A. P. } \\
\text { Leite }\end{array}$ & $\begin{array}{l}\text { Principais complicações } \text { da } \\
\text { circulação extracorpórea em } \\
\text { cirurgias cardíacas em um } \\
\text { hospital da região norte. }\end{array}$ & Saber científico & 3 \\
\hline 2013 & $\begin{array}{l}\text { Caroline Dienstmann; } \\
\text { Rita Catalina Aquino } \\
\text { Caregnato. }\end{array}$ & $\begin{array}{l}\text { Circulação extracorpórea em } \\
\text { cirurgia cardíaca: um campo } \\
\text { de trabalho para o enfermeiro. }\end{array}$ & Revista SOBECC & 4 \\
\hline 2017 & $\begin{array}{l}\text { Lara Morgana dos Reis } \\
\text { Freitas; Lia Pinto Cunha } \\
\text { Borges dos Santos; } \\
\text { Jeffchandler Belém De } \\
\text { Oliveira. }\end{array}$ & $\begin{array}{l}\text { Circulação extracorpórea e } \\
\text { desiquilíbrio hidroeletrolítico. }\end{array}$ & Journal Health NPEPS & 5 \\
\hline 2018 & Karen Saori Kakihara. & $\begin{array}{l}\text { Validação de um guia de boas } \\
\text { práticas para o cuidado } \\
\text { realizado pelo enfermeiro ao } \\
\text { paciente em circulação } \\
\text { extracorpórea. }\end{array}$ & $\begin{array}{l}\text { Trabalho de conclusão de } \\
\text { curso II (INT5182) } \\
\text { Curso de Graduação } \\
\text { Enfermagem } \\
\text { Universidade Federal } \\
\text { Santa Catarina. }\end{array}$ & 6 \\
\hline 2019 & $\begin{array}{l}\text { Gisela Lima; Manuel } \\
\text { Cuervo. }\end{array}$ & $\begin{array}{lrr}\text { Mecanismo da } & \text { Circulação } \\
\text { Extracorpórea } & \text { e } & \text { Eventos } \\
\text { Neurológicos } & \text { em } & \text { Cirurgia } \\
\text { Cardíaca. } & & \end{array}$ & $\begin{array}{lr}\text { Revista da } & \text { Sociedade } \\
\text { Portuguesa } & \text { de } \\
\text { Anestesiologia. } & \end{array}$ & 7 \\
\hline
\end{tabular}


Research, Society and Development, v. 10, n. 3, e33510310987, 2021

(CC BY 4.0) | ISSN 2525-3409 | DOI: http://dx.doi.org/10.33448/rsd-v10i3.10987

\begin{tabular}{|c|c|c|c|c|}
\hline 2011 & $\begin{array}{l}\text { Alessandra Renata Targa } \\
\text { Longo. }\end{array}$ & $\begin{array}{l}\text { Sistemas de aquecimento } \\
\text { cutâneo para prevenção de } \\
\text { hipotermia em cirurgia } \\
\text { cardíaca: revisão sistemática. }\end{array}$ & $\begin{array}{l}\text { Dissertação de mestrado da } \\
\text { Universidade de São Paulo }\end{array}$ & 8 \\
\hline 2016 & $\begin{array}{l}\text { Diego da Silva Neves; } \\
\text { Salete Maria de Fátima } \\
\text { Silqueira; Valéria Cristina } \\
\text { da Silva; Wágner do } \\
\text { Nascimento Carvalho. }\end{array}$ & $\begin{array}{l}\text { A atuação do enfermeiro } \\
\text { perfusionista na cirúrgica } \\
\text { cardíaca. }\end{array}$ & Revista Saúde & 9 \\
\hline 2013 & Carla Helena Pereira. & 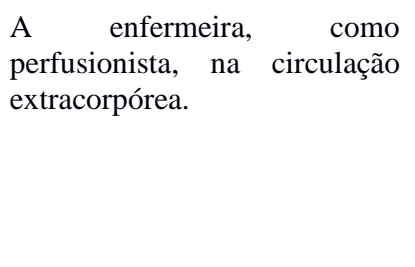 & $\begin{array}{l}\text { Trabalho de Conclusão } \\
\text { apresentado à Universidade } \\
\text { Federal do Rio Grande do } \\
\text { Sul como requisito parcial } \\
\text { para a conclusão do Curso } \\
\text { de Graduação em } \\
\text { Enfermagem. }\end{array}$ & 10 \\
\hline 2018 & 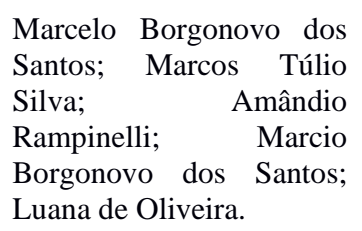 & $\begin{array}{l}\text { Comparação dos resultados } \\
\text { iniciais entre cirurgias de } \\
\text { revascularização do miocárdio } \\
\text { com e sem circulação } \\
\text { extracorpórea. }\end{array}$ & $\begin{array}{l}\text { Arquivo } \quad \text { Catarinense } \\
\text { Medicina. }\end{array}$ & 11 \\
\hline 2012 & \begin{tabular}{lrr} 
Camila & Sartorio; & Daniele \\
Cristina & Rocha & Lima; \\
Flaviana & de & Castro \\
Metello; Olga & Carla \\
Ribeiro Costa & Soares; \\
Valéria Cristina & Moreira \\
\multicolumn{2}{l}{ de Medeiros Mota; Flávia } \\
\multicolumn{2}{l}{ Silva de Souza. }
\end{tabular} & $\begin{array}{l}\text { Atuação do enfermeiro frente } \\
\text { aos efeitos da circulação } \\
\text { extracorpórea }\end{array}$ & Enfermagem Brasil & 12 \\
\hline 2016 & $\begin{array}{c}\text { Marco Aurélio; Pereira da } \\
\text { Silva. }\end{array}$ & $\begin{array}{lcr}\text { Relevância } & \text { da proteção } \\
\text { miocárdica } & \text { durante } & \text { a } \\
\text { circulação } & \text { extracorpórea } & \text { em } \\
\text { Porto Velho, Rondônia } & \end{array}$ & $\begin{array}{l}\text { Tese apresentada } \\
\text { Sociedade Brasileira de } \\
\text { Terapia Intensiva. }\end{array}$ & 13 \\
\hline 2012 & $\begin{array}{lcc}\text { Fernanda } & \text { Gaspar } & \text { Torrati; } \\
\text { Rosana } & \text { Ap. } & \text { Spadoti } \\
\text { Dantas. } & & \end{array}$ & $\begin{array}{l}\text { Circulação extracorpórea e } \\
\text { complicações no período pós- } \\
\text { operatório imediato de } \\
\text { cirurgias cardíacas. }\end{array}$ & Acta Paulista Enfermagem & 14 \\
\hline
\end{tabular}

Fonte: Autor (2020).

Contudo, no Quadro 2, mostra que houve uma constância nas publicações a partir de 2011 a 2019, apresentamos os desenhos dos estudos, objetivos e principais resultados. 
Quadro 2. Descrição dos estudos incluídos na revisão integrativa.

\begin{tabular}{|c|c|c|c|c|}
\hline Ordem & Título & Tipo de artigo & Objetivo do estudo & Conclusão/Resultados \\
\hline 1 & $\begin{array}{l}\text { Análise da variação de } \\
\text { eletrólitos após cirurgia } \\
\text { cardíaca com uso de } \\
\text { circulação extracorpórea } \\
\text { (Abreu, Thiago Titonel, } \\
\text { 2014) }\end{array}$ & $\begin{array}{l}\text { Revisão da } \\
\text { literatura. }\end{array}$ & $\begin{array}{l}\text { A circulação extracorpórea } \\
\text { proporciona mudanças no } \\
\text { equilíbrio dos eletrólitos, com } \\
\text { depleção, impactando sobretudo } \\
\text { nos níveis sérico de potássio, } \\
\text { cálcio, magnésio, fosfato. } \\
\text { Fenômeno presente mesmo com } \\
\text { a utilização de suplementação } \\
\text { desses eletrólitos. Sendo, } \\
\text { portanto, necessário avaliar a } \\
\text { ocorrência de alterações } \\
\text { eletrolíticas em pacientes } \\
\text { submetidos à CEC. }\end{array}$ & $\begin{array}{c}\text { Não se pode, entretanto, } \\
\text { distinguir com exatidão quais } \\
\text { sãos os efeitos específicos das } \\
\text { alterações eletrolíticas em } \\
\text { virtude da presença } \\
\text { concomitante da resposta } \\
\text { inflamatória Estudos } \\
\text { adequados são necessários para } \\
\text { se mapear esses efeitos } \\
\text { deletérios de modo a permitir a } \\
\text { elaboração de protocolos mais } \\
\text { precisos. }\end{array}$ \\
\hline 2 & $\begin{array}{c}\text { Determinantes de } \\
\text { Complicações } \\
\text { Neurológicas no Uso da } \\
\text { Circulação Extracorpórea } \\
\text { (CEC) (Barbosa, Natia de } \\
\text { Freitas; Caedinelli, Danilo } \\
\text { Martins; Ercole, Flávia } \\
\text { Falci, 2010) }\end{array}$ & $\begin{array}{l}\text { Revisão } \\
\text { Integrativa }\end{array}$ & $\begin{array}{l}\text { Identificar fatores de risco } \\
\text { inerentes à CEC e intervenções } \\
\text { que minimizem a incidência e } \\
\text { gravidade das lesões, } \\
\text { considerando o grau de } \\
\text { segurança deste procedimento. }\end{array}$ & $\begin{array}{l}\text { Novos estudos fisiopatológicos } \\
\text { são necessários para consolidar } \\
\text { essa área do conhecimento e } \\
\text { permitir o surgimento de } \\
\text { técnicas capazes de reduzir a } \\
\text { incidência e a extensão das } \\
\text { lesões neurológicas. }\end{array}$ \\
\hline 3 & $\begin{array}{c}\text { Principais complicações da } \\
\text { circulação extracorpórea } \\
\text { em cirurgias cardíacas em } \\
\text { um Hospital da Região } \\
\text { Norte. } \\
\text { BARROS, Sandy Ribeiro; } \\
\text { BANDEIRA, Michele } \\
\text { Matias; LEITE, Jandra } \\
\text { Cibele R. A. P. 2019) }\end{array}$ & $\begin{array}{l}\text { Pesquisa } \\
\text { quantitativa e } \\
\text { descritiva. }\end{array}$ & $\begin{array}{c}\text { Analisar as principais } \\
\text { complicações em pacientes } \\
\text { submetidos a cirurgias cardíacas } \\
\text { utilizando a circulação } \\
\text { extracorpórea. }\end{array}$ & $\begin{array}{l}\text { Para a equipe de enfermagem, } \\
\text { o conhecimento das } \\
\text { complicações no pós- } \\
\text { operatório de cirurgia cardíaca } \\
\text { com o uso da CEC facilita a } \\
\text { implementação de um plano de } \\
\text { cuidados adequado, prevenindo } \\
\text { maiores complicações, } \\
\text { sequelas e até mesmo óbitos. }\end{array}$ \\
\hline 4 & $\begin{array}{c}\text { Circulação extracorpórea } \\
\text { em cirurgia cardíaca: um } \\
\text { campo de trabalho para o } \\
\text { enfermeiro. } \\
\text { (DIENSTMANN, } \\
\text { Caroline; CAREGNATO } \\
\text { Rita Catalina Aquino. } \\
\text { 2013). }\end{array}$ & $\begin{array}{l}\text { Relato de } \\
\text { experiência, } \\
\text { com abordagem } \\
\text { qualitativa. }\end{array}$ & $\begin{array}{l}\text { Refletir sobre a função do } \\
\text { perfusionista nas cirurgias } \\
\text { cardíacas, desvelando um } \\
\text { campo de trabalho para a } \\
\text { enfermagem. }\end{array}$ & $\begin{array}{c}\text { Este relato de experiência } \\
\text { permitiu fazer uma reflexão } \\
\text { sobre a atuação do } \\
\text { perfusionista nas cirurgias } \\
\text { cardíacas, desvelando um } \\
\text { campo de atuação ainda pouco } \\
\text { explorado para a enfermagem. }\end{array}$ \\
\hline 5 & $\begin{array}{c}\text { Circulação extracorpórea e } \\
\text { desiquilíbrio } \\
\text { hidroeletrolítico. } \\
\text { (FREITAS, Lara Morgana } \\
\text { dos Reis; SANTOS, Lia } \\
\text { Pinto Cunha Borges dos; } \\
\text { OLIVEIRA, Jeffchandler } \\
\text { Belém De. 2017). }\end{array}$ & $\begin{array}{l}\text { Ensaio teórico } \\
\text { reflexivo. }\end{array}$ & $\begin{array}{l}\text { Realçar aspectos da circulação } \\
\text { extracorpórea relacionado ao } \\
\text { desiquilíbrio hidroeletrolítico. }\end{array}$ & $\begin{array}{l}\text { Verifica-se que os pacientes } \\
\text { submetidos a CEC ficam } \\
\text { sujeitos a sofrer com efeitos } \\
\text { colaterais que levam a um } \\
\text { efetivo desequilíbrio } \\
\text { hidroeletrolítico, sendo } \\
\text { essencial que haja um } \\
\text { monitoramento contínuo de seu } \\
\text { estado, no período intra e pós- } \\
\text { operatório. }\end{array}$ \\
\hline 6 & $\begin{array}{l}\text { Validação de um guia de } \\
\text { boas Práticas para o } \\
\text { cuidado realizado pelo } \\
\text { enfermeiro ao paciente em } \\
\text { circulação extracorpórea. } \\
\text { (KAKIHARA, Karen } \\
\text { Saori. 2018). }\end{array}$ & $\begin{array}{l}\text { Estudo } \\
\text { metodológico } \\
\text { com abordagem } \\
\text { quantitativa, } \\
\text { descritiva. }\end{array}$ & $\begin{array}{l}\text { Realizar a validação de } \\
\text { conteúdo por meio dos juízes } \\
\text { enfermeiros, o guia de boas } \\
\text { práticas construído para o } \\
\text { cuidado, realizado pelo } \\
\text { enfermeiro ao paciente em } \\
\text { circulação extracorpórea no } \\
\text { período transoperatório. }\end{array}$ & $\begin{array}{c}\text { Podemos avaliar por meio dos } \\
\text { testes estatísticos, a } \\
\text { consistência interna de Alfa de } \\
\text { Crombach e o Índice de } \\
\text { Validade de Conteúdo (IVC), } \\
\text { que o instrumento se mostrou } \\
\text { satisfatório para os juízes. O } \\
\text { que demonstra que os } \\
\text { cuidados, justificativas e } \\
\text { referências de cada um dos } 54 \\
\text { itens do instrumento, foram } \\
\text { considerados válidos. }\end{array}$ \\
\hline
\end{tabular}




\begin{tabular}{|c|c|c|c|c|}
\hline 7 & $\begin{array}{c}\text { Mecanismo da Circulação } \\
\text { Extracorpórea e Eventos } \\
\text { Neurológicos em Cirurgia } \\
\text { Cardíaca. } \\
\text { (LIMA, Gisela; CUERVO, } \\
\text { Manuel Cuervo. 2019) }\end{array}$ & $\begin{array}{l}\text { Revisão de } \\
\text { literatura. }\end{array}$ & $\begin{array}{l}\text { Descrever o desenvolvimento e } \\
\text { mecanismo da circulação } \\
\text { extracorpórea e as principais } \\
\text { complicações neurológicas } \\
\text { associadas à cirurgia cardíaca, } \\
\text { incluindo mecanismos } \\
\text { fisiopatológicos e possíveis } \\
\text { medidas de prevenção. }\end{array}$ & $\begin{array}{l}\text { A circulação extracorpórea } \\
\text { progrediu a passos firmes e } \\
\text { seguros ao longo destas últimas } \\
\text { décadas, desde a sua conceção } \\
\text { por Gibbon, em 1953. Apesar } \\
\text { da sua evolução e de todos os } \\
\text { procedimentos efetuados na } \\
\text { tentativa de minorar as } \\
\text { complicações, }\end{array}$ \\
\hline 8 & $\begin{array}{l}\text { Sistemas de aquecimento } \\
\text { cutâneo para prevenção de } \\
\text { hipotermia em cirurgia } \\
\text { cardíaca: revisão } \\
\text { sistemática. } \\
\text { (LONGO, Alessandra } \\
\text { Renata Targa. 2011). }\end{array}$ & $\begin{array}{c}\text { Revisão } \\
\text { Sistemática. }\end{array}$ & $\begin{array}{l}\text { Avaliar as evidências } \\
\text { disponíveis na literatura sobre o } \\
\text { qualé o sistema de aquecimento } \\
\text { cutâneo mais eficaz para a } \\
\text { prevenção da hipotermia, no } \\
\text { paciente submetido à cirurgia } \\
\text { cardíaca, sem circulação } \\
\text { extracorpórea, no período intra } \\
\text { operatório. }\end{array}$ & $\begin{array}{l}\text { Compete a esse profissional o } \\
\text { planejamento de intervenções } \\
\text { direcionadas para a melhoria da } \\
\text { qualidade do cuidado de } \\
\text { enfermagem e promovam a } \\
\text { segurança do paciente. }\end{array}$ \\
\hline 9 & $\begin{array}{l}\text { A atuação do enfermeiro } \\
\text { perfusionista na cirúrgica } \\
\text { cardíaca. } \\
\text { (NEVES, Diego da Silva et } \\
\text { al. 2016). }\end{array}$ & $\begin{array}{l}\text { Relato de } \\
\text { experiência. }\end{array}$ & $\begin{array}{l}\text { Relatar a experiência de um } \\
\text { enfermeiro perfusionista e suas } \\
\text { principais atribuições na } \\
\text { cirurgia cardíaca com } \\
\text { circulação extracorpórea. }\end{array}$ & $\begin{array}{l}\text { Com o avanço das tecnologias } \\
\text { e técnicas na área de cirurgia } \\
\text { cardiovascular, surgiu a } \\
\text { oportunidade da perfusão para } \\
\text { o profissional enfermeiro. } \\
\text { Considerando que seja um } \\
\text { desafio por se tratar de um } \\
\text { procedimento complexo que } \\
\text { exige além da capacitação, uma } \\
\text { interação intensa com a equipe } \\
\text { escalada para participar da } \\
\text { cirurgia e principalmente com } \\
\text { o cirurgião que está realizando } \\
\text { o procedimento. }\end{array}$ \\
\hline 10 & $\begin{array}{c}\text { A enfermeira, como } \\
\text { perfusionista, na circulação } \\
\text { extracorpórea. } \\
\text { (PEREIRA, Carla Helena. } \\
\text { 2013) }\end{array}$ & $\begin{array}{l}\text { Estudo de caso } \\
\text { com abordagem } \\
\text { exploratória. }\end{array}$ & $\begin{array}{l}\text { Conhecer a atuação do } \\
\text { enfermeiro na circulação } \\
\text { extracorpórea. }\end{array}$ & $\begin{array}{l}\text { Concluindo, foi possível } \\
\text { evidenciar o diferencial do } \\
\text { Perfusionista em possuir a } \\
\text { formação de Enfermagem, não } \\
\text { só pelo conhecimento } \\
\text { técnico/biológico que tem } \\
\text { acesso durante o curso, mas } \\
\text { essencialmente por trabalhar o } \\
\text { cuidado como base para toda a } \\
\text { sua assistência. }\end{array}$ \\
\hline 11 & $\begin{array}{l}\text { Comparação dos resultados } \\
\text { iniciais entre cirurgias de } \\
\text { revascularização do } \\
\text { miocárdio com e sem } \\
\text { circulação extracorpórea. } \\
\text { (SANTOS, Marcelo } \\
\text { Borgonovo dos et al. } \\
\text { 2018). }\end{array}$ & $\begin{array}{l}\text { Estudo } \\
\text { observacional } \\
\text { transversal } \\
\text { retrospectivo. }\end{array}$ & $\begin{array}{l}\text { Comparar os resultados iniciais } \\
\text { em grupos de pacientes } \\
\text { submetidos à cirurgia de } \\
\text { revascularização do miocárdio } \\
\text { com e sem circulação } \\
\text { extracorpórea }\end{array}$ & $\begin{array}{l}\text { A cirurgia de revascularização } \\
\text { sem CEC não demonstrou } \\
\text { resultados superiores de morbi- } \\
\text { mortalidade inicial em relação } \\
\text { aos pacientes submetidos à } \\
\text { cirurgia com CEC, indicando } \\
\text { que ambas as técnicas são } \\
\text { igualmente eficientes e } \\
\text { seguras. }\end{array}$ \\
\hline 12 & $\begin{array}{c}\text { Atuação do enfermeiro } \\
\text { frente aos efeitos da } \\
\text { circulação extracorpórea } \\
\text { (SARTORIO, Camila et al. } \\
\text { 2012). }\end{array}$ & $\begin{array}{l}\text { Revisão da } \\
\text { literatura, de } \\
\text { natureza } \\
\text { descritiva e } \\
\text { exploratória. }\end{array}$ & $\begin{array}{c}\text { Identificar as principais } \\
\text { complicações ocasionadas pela } \\
\text { CEC e discutir a conduta do } \\
\text { enfermeiro na minimização dos } \\
\text { efeitos indesejáveis causados } \\
\text { pela CEC. }\end{array}$ & $\begin{array}{l}\text { Conclui-se que as cirurgias que } \\
\text { utilizam a CEC podem levar a } \\
\text { complicações que, se não } \\
\text { forem evitadas ou } \\
\text { minimizadas, poderão } \\
\text { desenvolver alterações severas } \\
\text { e de difícil reversão. Das } \\
\text { complicações evidenciadas a } \\
\text { mais comum está relacionada à } \\
\text { mecânica ventilatória. }\end{array}$ \\
\hline
\end{tabular}




\begin{tabular}{|c|c|c|c|c|}
\hline 13 & $\begin{array}{l}\text { Relevância da proteção } \\
\text { miocárdica durante a } \\
\text { circulação extracorpórea } \\
\text { em porto velho, Rondônia. } \\
\text { (Silva, Marco Aurélio } \\
\text { Pereira da. 2016). }\end{array}$ & $\begin{array}{c}\text { Revisão } \\
\text { Integrativa de } \\
\text { Literatura. }\end{array}$ & $\begin{array}{c}\text { O presente trabalho busca } \\
\text { sintetizar o conhecimento } \\
\text { produzido na literatura } \\
\text { científica acerca da importância } \\
\text { da proteção miocárdica no } \\
\text { decorrer do procedimento de } \\
\text { cirurgia cardíaca e, ainda, } \\
\text { abordar as variantes de proteção } \\
\text { miocárdica. }\end{array}$ & $\begin{array}{l}\text { A preocupação com a proteção } \\
\text { miocárdica sempre deverá ser } \\
\text { um dos pontos mais } \\
\text { importantes desde o momento } \\
\text { da decisão de que o paciente } \\
\text { será submetido à cirurgia } \\
\text { cardíaca, visando melhorar a } \\
\text { qualidade de vida, } \\
\text { principalmente daqueles } \\
\text { pacientes que apresentam } \\
\text { algum grau de deficiência do } \\
\text { trabalho do músculo cardíaco. }\end{array}$ \\
\hline 14 & $\begin{array}{l}\text { Circulação extracorpórea e } \\
\text { complicações no período } \\
\text { pós-operatório imediato de } \\
\text { cirurgias cardíacas. } \\
\text { (TORRATIL, Fernanda } \\
\text { Gaspar; DANTAS, Rosana } \\
\text { Ap. Spadoti. 2012). }\end{array}$ & $\begin{array}{l}\text { Estudo de } \\
\text { natureza } \\
\text { quantitativa, } \\
\text { descritivo e } \\
\text { correlacional } \\
\text { com } 83 \\
\text { pacientes } \\
\text { adultos } \\
\text { divididos em } \\
\text { dois grupos de } \\
\text { acordo com o } \\
\text { tempo de CEC. }\end{array}$ & $\begin{array}{l}\text { Comparar a frequência de } \\
\text { complicações apresentadas } \\
\text { pelos pacientes, durante o pós- } \\
\text { operatório imediato (POI), de } \\
\text { cirurgias cardíacas de acordo } \\
\text { com o tempo de circulação } \\
\text { extracorpórea (CEC). }\end{array}$ & $\begin{array}{l}\text { A maioria das complicações } \\
\text { ocorridas no POI apresentou } \\
\text { frequência semelhante para os } \\
\text { pacientes, independente do } \\
\text { tempo de CEC. }\end{array}$ \\
\hline
\end{tabular}

Fonte: Autor (2020).

A partir dos resultados encontrados nos artigos selecionados, foi possível analisar os conteúdos e abordar os objetivos propostos pelo estudo. Iniciamos então com uma abordagem sobre a cirurgia cardíaca.

A cirurgia cardíaca tem sido possível devido aos avanços, nestes avanços como principal evolução se dá pelo desenvolvimento da circulação extracorpórea, esta fornece um campo com visibilidade direta do coração. Estudos relatam que a CEC tem a função de suporte circulatório e respiratório junto ao controle de temperatura para que acha facilidade na correção cirúrgica do coração ou de grandes vasos (Freitas, Santos \& Oliveira, 2017).

$\mathrm{Na}$ CEC utiliza-se um circuito extracorpóreo para fornecer suporte fisiológico, a qual o sangue venoso é drenado para um reservatório oxigenado e enviado de volta para corpo usando uma bomba. No entanto, a CEC surge como um procedimento único e inovador em 6 de maio de 1953, no Hospital Jefferson, localizado nos Estados Unidos, neste local foi realizado a primeira cirurgia cardíaca utilizando o método coração-pulmão artificial, idealizadores foram o John Gibbon e sua esposa Mary Gibbon, estes construíram tal sistema capaz de suprir temporariamente as necessidades metabólicas de um ser humano. Já no Brasil Hugo Felipozzi, em São Paulo criou em 1955 uma oficina para estudos experimentais no Hospital das Clínicas da Universidade Federal de São Paulo para iniciar a construção e fabricação de equipamentos para a padronização dos aparelhos da circulação extracorpórea (Abreu, 2014; Silva, 2016).

Lima e Cuervo (2019) e Sartorio et al (2012), em seus estudos explica que a CEC constitui de um conjunto de máquinas, aparelhos, tubos e técnicas que podem substituir temporariamente as funções de bomba do coração e ventilatória dos pulmões, isso em decorrência de evento cirúrgico a qual estes órgãos encontram-se. Este autor explica ainda que tal equipamento, possui dois reservatórios, onde o reservatório venoso tem como função receber o sangue proveniente da drenagem venoso, e o reservatório de cardiotomia tem a função de receber o sangue proveniente do campo operatório, recuperado por aspiração. Ainda possui um oxigenador que fica acoplado a um permutador de calor e entre o oxigenador e a cânula arterial, é instalado um filtro de linha arterial.

A CEC permite que lesões cardíacas sejam restauradas, dando ao cirurgião a oportunidade de trabalhar de forma segura e realizar a correção de defeitos, considerados impossíveis em outras formas. Esta técnica deverá ser realizada de forma perfeita de entrosamento de toda equipe envolvida, para que nenhum detalhe escape à observação correta, evitando, colocar 
em risco a vida do paciente ou sua integridade física e mental. Procedimento considerado de alta complexidade, onde os profissionais, realize-o com segurança, precisam deter de conhecimentos profundos, de forma que as decisões sejam automáticas e imediatas. Para adquirir estas habilidades, se faz necessário os conhecimentos teóricos sólidos e treinamento exaustivo (Lima \& Cuervo, 2019; Torratil \& Dantas, 2012).

Contudo, estudos corroboram, que com esta nova tecnologia vieram outros conhecimentos acerca da fisiologia da circulação, como as reações do organismo as agressões cirúrgicas e ao domínio do metabolismo e da compreensão da homeostase, que se caracteriza por um sistema complexo de auto-regulação, para manter os múltiplos paramentos vitais. Com isso, esse processo resulta em catabolitos, calor, ácidos, água, gás carbônico e outros elementos químicos que precisam ser transformados e ou eliminados. Este estudo ainda relata que estes avanços da Medicina e da pesquisa, permitiu que não se restringisse apenas ao sistema cardiovascular, mas também beneficiar a compreensão das variações dos equilíbrios ácidobásico e hidrosalino, indispensáveis para a estabilidade destes sistemas durante a Circulação Extracorpórea (Lima \& Cuervo, 2019).

Através destes conceitos foi possível compreender o campo da fisiologia, as consequências dos desequilíbrios ácidobasico e hidrosalino em estados críticos, incluindo o choque cardiogênico, o choque séptico, tornando fundamental para equipe multidisciplinar que realiza a cirurgia cardíaca. Pois neste momento, a qual a circulação sanguínea e a respiração mantêm-se artificialmente, a fisiologia orgânica deve ser monitorada e ajustada para que fique o mais próximo dos parâmetros da normalidade. O conhecimento acerca das trocas gasosas é fundamental para manter o paciente em condições perfeitas de fornecimento de oxigênio e substratos, com a retirada de gás carbônico e catabolitos da melhor forma possível (Barros, Bandeira \& Leite, 2019).

Barbosa, Bandeira e Leite (2019), em seus estudos, identifica um ponto importante a qual esta pesquisa corrobora, mostrando que o controle da temperatura durante o processo operatório, tanto no período de hipotermia, se faz necessário para diminuir o metabolismo. A hipotermia é um procedimento que transcende a nossa expectativa, este possibilita parar totalmente a circulação por uma hora ou mais, e posteriormente ao aquecimento, ver o paciente voltar ao seu estado de metabolismo homeotérmico e à vida.

Segundo Lima e Cuervo (2019), a utilização da circulação extracorpórea também pode provocar alterações neurológicas, para evitá-la este estudo relata que os glicocorticoides, anestésicos, barbitúricos, oxigenadores de membrana, câmeras hiperbáricas, hipotermia, hemofiltração, circuitos revestidos de heparina e a manutenção da temperatura, do PH e da volemia. Contudo, o estudo cita que a hipotermia permite aumentar o tempo de parada cardíaca, diminuindo a urilização de oxigênio no cérebro, evitando dano neurológico. Mas este autor também relata que a hipotermia favorece a hemoconcentração e o aumento da viscosidade sanguínea.

Ressaltando, que a hipotermia também é utilizada como forma de proteção neurológica, onde a diminuição da temperatura reduz consideravelmente o fluxo sanguíneo cerebral e o consumo metabólico cerebral de oxigênio. No entanto, temperaturas quando mantidas em $28^{\circ} \mathrm{C}$, pode está relacionada à uma menor incidência de disfunção cognitiva, quando comparadas com temperaturas elevadas. Isso quando relacionado com a hipotermia moderada, mantendo a pressão-fluxo inalterada. Nesta técnica de hipotermia moderada, ocorre também um aumento da solubilidade do dióxido de carbono (CO2), assim, as baixas temperaturas diminuem a $\mathrm{PaCO} 2$ (pressão parcial de gás carbônico) ocorrendo o aumento do pH arterial. Com isso existem dois tipos de manter o equilíbrio ácido-base durante a CEC. Anteriormente utilizava-se o CO2 na máquina de CEC para manter um $\mathrm{pH}$, método conhecido como pH-stat, mas atualmente não ocorre a correção da PaCO2, método conhecido como alfa-stat, e sim, deixando o $\mathrm{pH}$ várias livremente com a temperatura, considerando como um estado mais fisiológico (Lima \& Cuervo, 2019; Santos et al., 2018). 
Estudos realizados por Kakiara (2018) e Neves et al (2016), corrobora que a hipotermia se faz necessária para redução do consumo de oxigênio do organismo, ocorre pelo baixo nível da atividade das reações químicas do metabolismo. Este procedimento de hipotermia é induzida pela circulação de água com gelo no permutador de calor acoplado ao oxigenador. As modificações de temperatura devem ser monitoradas para reduzir o desprendimento de gases, através de variações térmicas bruscas.

Na literatura foi possível compreender a necessidade do controle da temperatura corporal, evitando hipertermia, controlando o ritmo cardíaco, o estado equilíbrio ácido-base e hidroeletrolítico.

\section{Considerações Finais}

Com este estudo foi possível compreender a importância da utilização da hipotermia na CEC, para reduzir danos ao paciente, diminuindo a ocorrência de complicações neurológicas. Portanto, é importante ressaltar que o estudo teve como principal objetivo compreender a ação da hipotermia no paciente em uso da CEC.

Para compreensão deste estudo, é necessário entender o que seria a hipotermia, que seria um procedimento com a aplicação terapêutica do frio, realizando a diminuição da temperatura corporal abaixo de $35^{\circ} \mathrm{C}$, esta não poderá chegar a $32^{\circ} \mathrm{C}$. Esta terapêutica consiste em diminuir a demanda de oxigênio, exercendo a proteção dos órgãos vitais (cérebro, coração, rins), ainda diminui a frequência cardíaca e melhora a perfusão miocárdica (Barbosa, Cardinelli \& Ercole, 2010; Longo, 2011).

Este estudo permitiu uma reflexão a atuação nas cirurgias cardíaca, difundindo o conhecimento do uso da hipotermia neste tipo de cirurgia. Concluindo que apesar de muitos avanços tecnológicos, a hipotermia ainda persiste em não ser utilizada para evitar as complicações fisiopatológicas. Sendo assim, indispensável para descrever os mecanismos que são positivos utilizados na hipotermia.

\section{Referências}

Abreu, T. T. (2014). Análise da variação de eletrólitos após cirurgia cardíaca com uso de circulação extracorpórea. Monografia (Conclusão de Curso) Universidade Federal da Bahia, Faculdade de Medicina da Bahia, Salvador.

Barbosa, N. F., Cardinelli, D. M., \& Ercole, F. F. (2010). Determinantes de Complicações Neurológicas no Uso da Circulação Extracorpórea (CEC). Arquivo Brasileiro Cardiologia, 95(6): e151-e157.

Barros, S. R., Bandeira, M. M., \& Leite, J. R. A. P. (2019). Principais complicações da circulação extracorpórea em cirurgias cardíacas em um Hospital da Região Norte. Saber Científico, Porto Velho, 8(1), $103-110$.

Dienstmann, C., \& Caregnato, R. C. A. (2013). Circulação extracorpórea em cirurgia cardíaca: um campo de trabalho para o enfermeiro. Rev. SOBECC, 18(1): 35-43.

Freitas, L. M. R., Santos, L. C. B., \& Oliveira, J. B. (2017). Circulação extracorpórea e desiquilíbrio hidroeletrolítico. Journal Health NPEPS, 2 (1):285-297.

Hueb, T. O., \& Hueb, W. (2012). Revascularização do miocárdio com e sem circulação extracorpórea. Rev. Bras. Medicina, 70(1/2), 39-45. http://moreirajr.com.br/revistas.asp?fase=r003\&id_materia=5324.

Kakihara, K. S. (2018). Validação de um guia de boas práticas para o cuidado realizado pelo enfermeiro ao paciente em circulação extracorpórea. Trabalho de conclusão de curso, referente à disciplina: Trabalho de conclusão de curso II (INT5182) do Curso de Graduação em Enfermagem da Universidade Federal de Santa Catarina, como requisito parcial para obtenção do Grau de Enfermeiro. Florianópolis.

Lima, G., Cuervo, M. (2019). Mecanismo da Circulação Extracorpórea e Eventos Neurológicos em Cirurgia Cardíaca. Revista da sociedade portuguesa de anestesiologia. 28(1).

Longo, A. R. T. (2011). Sistemas de aquecimento cutâneo para prevenção da hipotermia em cirurgia cardíaca: revisão sistemática. Dissertação apresentada a Escola de Enfermagem de Ribeirão Preto da Universidade de São Paulo, para obtenção do título de mestre em ciências. Ribeirão Preto.

Neves, D. S. et al (2016). A atuação do enfermeiro perfusionista na cirúrgica cardíaca. Revista Saúde, 10(1).

Pereira, C. H. (2013). A enfermeira, como perfusionista, na circulação extracorpórea. Trabalho de Conclusão apresentado à Universidade Federal do Rio Grande do Sul como requisito parcial para a conclusão do Curso de Graduação em Enfermagem.

Santos, M. B. et al (2018). Comparação dos resultados iniciais entre cirurgias de revascularização do miocárdio com e sem circulação extracorpórea. Arquivos Catarinenses Medicina, 47(2):170-181. 
Research, Society and Development, v. 10, n. 3, e33510310987, 2021

(CC BY 4.0) | ISSN 2525-3409 | DOI: http://dx.doi.org/10.33448/rsd-v10i3.10987

Sartorio, C. et al (2012). Atuação do enfermeiro frente aos efeitos da circulação extracorpórea. Enfermagem Brasil. 11(2).

Silva, M. A. P. (2016). Relevância da proteção miocárdica durante a circulação extracorpórea em Porto Velho, Rondônia. Tese apresentada à Sociedade Brasileira de Terapia Intensiva, como requisito obrigatório para obtenção do título de Mestre em Terapia Intensiva. Porto Velho, RO.

Souza, M. H. L., \& Elias, D. O. (2006). Fundamentos da circulação extracorpórea. (2a ed.), Centro Editorial Alfa Rio. https://sbcec.com.br/br/images/blog/livromariahelena.pdf. Acesso em: 20 agost. 2020.

Torrati, F. G., \& Dantas, R. A. S. (2012). Circulação extracorpórea e complicações no período pós-operatório imediato de cirurgias cardíacas. Acta Paul Enferm.25(3):340-5. 